\title{
Determinates of depressive disorder among adult patients with cardiovascular disease at outpatient cardiac clinic Jimma University Teaching Hospital, South West Ethiopia: cross-sectional study
}

Halima Umer ${ }^{1}$, Alemayehu Negash², Mengesha Birkie ${ }^{3}$ and Asmare Belete ${ }^{3 *}$

\begin{abstract}
Background: Depression and heart disease are an important public-health problem. Depression is one of the most prevalent and disabling psychiatric disorders with more than three times increased risk among patients with cardiovascular disorders.

Objective: To identify the prevalence and associated factors of depressive disorder among adult patients with cardiovascular disease.

Methods: Institution based cross-sectional study design was used to conduct this study on 293 study participants attending an outpatient cardiac clinic at Jimma University Teaching Hospital. All eligible patients were recruited into the study consecutively. Depression was assessed using patient health questionnaire-9. The patient health questionnaire-9 had a total score of 27, from which 0-4: no/minimal depression, 5-9: mild depression, 10-14: moderately depression, 15-19: moderately severe depression and 20-27 severe depression. The data was feed into Epi-data version 3.1 and lastly exported to SPSS version 21 for analysis. Bivariate analysis was used to analyze the statistical association of covariates of interest with depressive disorder among patients with cardiovascular disease. Then, logistic regression analysis was used as a final model to control confounders. The strength of association was measured by a 95\% confidence interval.
\end{abstract}

Results: A total of 293 adult patients diagnosed with the cardiovascular disease were included in the study with $97 \%$ $(n=284)$ of response rate, $47.2 \%(n=134)$ males and $52.8 \%(n=150)$ females, making female to a male ratio around 1.1:1. The prevalence of depression was $52.8 \%(n=150 / 284)$. Out of the subjects with depression $52.67 \%(n=79)$, $36.0 \%(n=54)$ and $11.33 \%(n=17)$ were mild, moderate and severe depression, respectively. Variables such as employed, unemployed, physical activity, current cigarette user and poor social support were independently associated with depression in the final model.

Conclusions: In this study, depression was found to be highly prevalent psychiatric comorbidity in adult cardiovascular disease patients.

Keywords: Depression, Cardiovascular disease, Ethiopia, Determinants, Patient health questionnaire-9

\footnotetext{
*Correspondence: yasmarebel@yahoo.com

${ }^{3}$ Department of Psychiatry, College of Medicine and Health Sciences,

Wollo University, Dessie, Ethiopia

Full list of author information is available at the end of the article
} 


\section{Background}

Non-communicable diseases (NCD) currently account $60 \%$ of all deaths and $48 \%$ of the disability-adjusted life years (DALYs) worldwide, $40 \%$ for communicable diseases. In 2008, four out of five NCD deaths occurred in low- and middle-income countries [1]. Mental disorders are a major contributor to the burden of disease in all regions of the world [2]. Mental health conditions are the leading cause of DALYs worldwide and account for 37\% of years of life lost (YLL) from NCDs [1].

According to the World Health Organization (WHO) report in 2011 unipolar depressive disorder is the third leading cause of disease burden worldwide. Mental disorders account for $25.3 \%$ and $33.5 \%$ of all years lived with a disability in low- and middle-income countries (LAMICs), respectively [3]. Mental illness is both a direct cause of mortality and a major risk factor for adverse health outcomes [4].

Depression is a substantial contributor to the global burden of disease. It affects people in all communities worldwide. In 2012, the WHO reported that depression is estimated to affect 350 million peoples [5]. Depression is a common mental disorder that has a clinical feature of depressed mood, loss of interest, reduced energy, feelings of guilt or low self-esteem, disturbance of sleep/appetite, and poor concentration, severe enough to cause severe impairment in important role function. At its worst, depression leads to suicide and is responsible for 1 million deaths due to suicide every year, which translates to 3000 suicide deaths every day [5].

World Health O report shows that 17.3 million people died from CVDs, contributing to $30 \%$ of all global deaths and this represents $50 \%$ of all death from NCD. In the same year, CVDs were the number one cause of death and deaths from these disorders in LAMIC constituted $80 \%[6,7]$. At the same time, it is estimated that about 19 million deaths occur annually from cardiovascular causes in lower-income countries [1, 6]. Mental health and cardiovascular diseases account for almost $70 \%$ of global economic losses [1].

The reported prevalence of major depression in patients with CAD is around $20 \%$, the prevalence of depression is among patient with CHD is very high (31-34\%) and is about three times more compared to that in the general population. Moreover, depression has been found to be a risk factor for the development of CAD and worsens its outcome when depression coexists with established CAD [19-21]. One-fifth of the patients with CHD attending outpatients and one-third of patients with congestive heart failure (CHF) have coexisting depression.
Depression in patients with CHD and CHF, however, is not recognized or not properly treated most of the time [8].

Quality of life of patients and their significant others are seriously affected by debilitating physical health, psychological distress, and diminished family role [9]. In patients undergoing coronary artery bypass graft (CABG), depression has been associated with longer hospitalization, poorer functional outcomes, more preoperative complications, higher rates of mortality and more symptomatic before and also after surgery $[10,11]$.

Effects of untreated depression that include: increased risk of a coronary event, decreased feelings of wellbeing and quality of life (QOL), decreased medication compliance and decreased risk factor modification. Can seriously increase the risks of further cardiac morbidity or mortality. Despite this knowledge; Physicians usually under-detect depression among cardiac patients; moreover, some of the cardiovascular drugs such as beta blockers may worsen the overall depressive symptoms $[12,13]$.

Factors associated with depression, most studies have found that younger patients and women were more likely to have the disorder in the context of CVD [16]. Poor social support, prior ACS, and in some cases, comorbid diabetes may also increase depression risk [14-16].

Many studies implicate depression among CAD patients to have the association with female sex, younger age, living situation, prior ACS, comorbid diabetes, HF severity class, low educational attainment, use of beta blockers, being housewives, unemployment status [14, 17-21]. Positive family history of depression, smoking, history of CHD/MI, social isolation/social support, NYHA CLASS classification system based on clinical severity and prognosis, antihypertensive medication [22, 23]. People with depression are 25 to $40 \%$ more likely to die from heart disease than people without depression [24].

Furthermore, several candidate biological and behavioral factors believed to be mechanisms through which depression could lead to cardiac events have been identified. Such as tricyclic antidepressants toxicity associated with cardiac risk factors. Potential behavior related mechanisms comprise dietary factors, sedentary lifestyle with lack of exercise, non-adherence to cardiac prevention and treatment regimens, lack of optimal social support, unhealthy lifestyles such as smoking, alcoholism and reduced functional capacity $[8,25]$.

Therefore, the fact that depression is a predictor of cardiac events among patients with CVD is has gained universal consensus although such information is lacking from countries such as Ethiopia. Furthermore, it was a well-known fact that comorbid depression increases 
negative events such as progression to arteriosclerosis, increased health care utilization and increased hospital readmissions before or after a cardiac event.

In spite of, evidence has suggested that depression can aggravate the course of multiple cardiovascular conditions, detection of depression in cardiac patient remains low. According to the principal investigator's knowledge, there is no study conducted in Ethiopia and the extent of the problem is not yet known. A better understanding of the extent of depression and associated factors is significant. This research will narrow this huge gap and will serve as a baseline for further researches to be undertaken on the subject matter.

\section{Methods and materials}

\section{Study setting and period}

The study was conducted in Jimma University Teaching Hospital (JUTH) outpatient cardiac clinic from October to November 2014. It has been running a public health care institution under the ministry of health by different names at a different time. Geographically, it is located in Jimma town $352 \mathrm{~km}$ south west of Addis Ababa. It provides service for at least 15 million populations residing in South-West Ethiopia. The cardiac clinic is one of the follow-up clinics giving service for a patient with chronic CVDs among other clinics that give service for patients with other chronic NCDs [26].

\section{Participants}

The study participants were all adult patients who had CVD age 18 years and above who came for follow-up at JUTH cardiac clinic during the study period. A total of 293 adult patients who had CVD were involved in the study. This study exclude, individual who seriously ill and were not able to give information, had a severe neuropsychiatric condition or language deficit that would preclude informed consent or valid assessment was excluded.

\section{Measurements}

The dependent variable was depression. The independent variables include socio-demographic characteristics such as age, sex, marital status, educational status, place of residence, income, empowerment, psychosocial, medication, clinical-related, behavioral and lifestyle factors.

\section{Data collection procedures and instruments}

Data were collected using interview-administered questionnaires. Questionnaires were abstracted and adapted from different literature sources and modified according to the local context. The questionnaire prepared by the English language, translate to Afan Oromo and Amharic language by the language department who is a native speaker for oromifa language and back translation also made. Depression was measured using patient health questionnaire nine (PHQ-9) which is a validated instrument in Ethiopia [27]. The PHQ-9 has a total score of 27, from which 0-4: no/minimal depression, 5-9: mild depression, 10-14: moderately depression, 15-19: moderately severe depression and 20-27 severe depression. Morisky medical adherence scale-8 (MMAS-8) was used to assess medication adherence which is a validated tool in Ethiopia to screening and monitoring in clinical practice to identify and monitor high-risk non-adherent patients [28].

Oslo 3-items social support scale (OSS-3) was used to measure the strength of social support [29]. Pretest of the questionnaire was carried out on $5 \%$ of respondents; whose socio-demographic factors are the same with those actual study participants; from the cardiac clinic in Jimma University Teaching Hospital (JUTH) those individuals not included in the main study differentiated by making a mark on their chart.

\section{Data processing, analysis, interpretation, and presentation} The quantitative data were entered into the computer by using Epi-data version 3.1 and lastly exported to SPSS version 21 for analysis. Bivariate analysis and multiple logistic regressions were used. Finally, variables had a P-value of less than .25 on binary logistic regression were entered into multivariable logistic regression. The strength of association was measured by $95 \%$ confidence interval and Then P-value $<.05$ considered as significantly associated variables with depression in the final model. The result was presented by frequency tables, graphs and discussed with previous study findings.

\section{Ethical consideration}

The ethical approval of the study was obtained from the Ethical Review Board of Jimma University, college of public health and Medical Sciences. Oral consent was obtained from the study participants. Patient those found to be severally depressed and suicidal risk appropriate intervention was done according to PHQ-9 score.

\section{Results \\ Socio-demographic characteristics}

A total of 293 adult patients diagnosed with CVD were included in the study with a response rate of $97 \%$ $(n=284)$. Out of the total respondents $47.2 \%(n=134)$ males and $52.8 \%(n=150)$ females with male-to-female ratio of 1.1:1. The age of the respondents ranged from 18 to 90 with a mean (SD) of 50.3 (17.13) years.

Nearly to two-third of (64.4\%) patients came from the rural area and nearly three-fourths of the respondents were married (76.0\%). About one-half of the total participants were illiterate $(53.2 \%)(\mathrm{n}=151)$. Regarding the 
occupation status, more than one half of the total of the respondents were farmers, $50.7 \%(n=144)$. Unemployed and housewives constituted 18\% $(\mathrm{n}=144)$ and $8.8 \%$ $(n=25)$ respectively. About $26.1 \%(n=74)$ of the study participants claimed to earn an annual average income of 10,800 birrs (Table 1 ).

Table 1 Socio-demographic characteristics of the respondents in Jimma University Teaching Hospital cardiac clinic, 2014$2015(n=284)$

\begin{tabular}{|c|c|c|c|}
\hline Characteristics & $\begin{array}{l}\text { Non } \\
\text { depressed } \\
\text { (PHQ-9<5) } \\
\mathrm{N}(\%)\end{array}$ & $\begin{array}{l}\text { Depressed } \\
\text { (PHQ-9 } \geq 5) \\
\mathrm{N}(\%)\end{array}$ & Total (\%) \\
\hline \multicolumn{4}{|l|}{ Sex } \\
\hline Male & $60(44.8)$ & $74(49.3)$ & $134(47.2)$ \\
\hline Female & $74(55.2)$ & $76(50.7)$ & $150(52.8)$ \\
\hline \multicolumn{4}{|l|}{ Age } \\
\hline $18-26$ & $17(12.7)$ & $17(11.3)$ & $34(12.0)$ \\
\hline $27-35$ & $15(11.2)$ & $21(14.0)$ & $36(12.7)$ \\
\hline $36-44$ & $20(14.9)$ & $13(8.7)$ & $33(11.6)$ \\
\hline $45-53$ & $22(16.4)$ & $25(16.7)$ & $47(16.5)$ \\
\hline-62 ref & $28(20.9)$ & $26(17.3)$ & $54(19.0)$ \\
\hline $63-71$ & $22(16.4)$ & $28(18.7)$ & $50(17.6)$ \\
\hline$\geq 72$ & $10(7.5)$ & $20(13.3)$ & $30(11.2)$ \\
\hline \multicolumn{4}{|l|}{ Marital status } \\
\hline Single & $20(14.9)$ & $13(8.7)$ & $33(11.6)$ \\
\hline Married & $105(78.4)$ & $111(74.0)$ & $216(76.0)$ \\
\hline Divorced/separate & $4(3.0)$ & $10(6.7)$ & $14(5.0)$ \\
\hline Widowed & $5(3.7)$ & $16(10.7)$ & $21(7.4)$ \\
\hline \multicolumn{4}{|l|}{ Educational status } \\
\hline Illiterate & $67(5.0)$ & $84(56.0)$ & $151(53.2)$ \\
\hline Able to read and write only & $20(14.9)$ & $36(24.0)$ & $56(19.7)$ \\
\hline Primary (1-8) & $28(20.9)$ & $19(12.7)$ & $47(16.5)$ \\
\hline Secondary (9-12) & $14(10.4)$ & $7(4.7)$ & $21(7.4)$ \\
\hline Tertiary $(+12)$ & $5(3.7)$ & $4(2.7)$ & $9(3.2)$ \\
\hline \multicolumn{4}{|l|}{ Occupational status } \\
\hline Unemployed & $16(11.9)$ & $35(23.3)$ & $51(18.0)$ \\
\hline Employed & $12(9.0)$ & $4(2.7)$ & $16(5.6)$ \\
\hline Farmer & $70(52.2)$ & $74(49.3)$ & $144(50.7)$ \\
\hline Merchant & $9(6.7)$ & $10(6.7)$ & $19(6.7)$ \\
\hline Retired & $7(5.2)$ & $5(3.3)$ & $12(4.2)$ \\
\hline Housewife & $11(8.2)$ & $14(9.3)$ & $25(8.8)$ \\
\hline Other & $9(6.7)$ & $8(5.3)$ & $17(6.0)$ \\
\hline \multicolumn{4}{|l|}{ Residence } \\
\hline Rural areas & $80(59.7)$ & $103(68.7)$ & $183(64.4)$ \\
\hline Urban areas & $54(40.3)$ & $47(31.3)$ & $101(35.6)$ \\
\hline \multicolumn{4}{|l|}{ Average annual income } \\
\hline 0-999 & $33(24.6)$ & $37(24.7)$ & $70(24.6)$ \\
\hline 1000-3599 & $32(23.9)$ & $37(24.7)$ & $69(24.3)$ \\
\hline $3600-10,799$ & $28(20.9)$ & $43(28.7)$ & $71(25.0)$ \\
\hline$\geq 10,800$ & $41(30.6)$ & $33(22.0)$ & $74(26.1)$ \\
\hline
\end{tabular}

Other employment status - student, daily labor and house servants

\section{Psychosocial and behavioral factors}

The majority (90.5\%) of respondents were living with their family and $6.3 \%(\mathrm{n}=18)$ were living alone. Only $24.4 \%$ $(\mathrm{n}=69)$ reported having good social support whereas $35.2 \%(n=100)$ claimed to have poor social support. Those who reported having almost satisfactory or moderate social support constituted $40.5 \%(n=115)$. About half of respondent (48.9\%) do not do moderate to vigorous activities for $30 \mathrm{~min}$ or more, at least 4 days in a week. Respondents who used that in their lifetime were $26.8 \%$ $(\mathrm{n}=76)$ and $8.8 \%(\mathrm{n}=25)$ were current use (Table 2$)$.

\section{Clinical characteristics and medication related factors Medication related factors}

The most prescribed drugs were diuretics in $75.0 \%$ $(\mathrm{n}=213)$; angiotensin-converting enzyme inhibitors in $55.3 \%(n=157)$ and beta-blockers in $45.2 \%(n=137)$. The rate of depression was higher at $77.3 \%(n=116)$ among participants who were taking diuretics. The majority of participants, $32.0 \%(n=91)$ were $\leq 1$ year of duration of treatment. The rate of depression is higher among those whose duration of treatment for CVD was greater than or equal to 5 years $30.7 \%(n=46)$. Nearly three-fourth of participants $(n=206)$ were adherence to CVD medication (Table 3).

\section{Clinical related factors}

The majority of participants were diagnosed with Hypertensive heart disease (HHD) constituting 35.9\% $(\mathrm{n}=102)$. Cases with CHD were found in $26.4 \%(\mathrm{n}=75)$ whereas patients with Valvular heart disease (VHD) were found in $12.3 \%(n=35)$. The highest rate of depression was found among HHD 36.0\% $(n=54)$ and the lowest in other diagnostic groups of CVD (Corpulmonary, acute rheumatic fever, congenital heart disease, pericarditis and CHF due to thyrotoxicosis) classification $6.0 \%(n=9)$. Among depressed $30.0 \%(n=45)$ were the age of onset of CVD above 60 years. Close to one-third (30\%) of the patients have been ill with CVD for the duration of 1-3 years.

Majority of participants $87.3 \%(n=248)$ had no family history of mental illness. From those who had a family history of mental illness $(55.6 \% ; n=20 / 36)$ were more depressed as compared to those no history of depression (44.4\%; $n=16 / 36)$. Two hundred seventy (95.1\%) of participants had no comorbid diabetes mellitus. Among those who had comorbid diabetes mellitus, most had depressed mood $(64.3 \% ; \mathrm{n}=9 / 14)$ as compared to that non-comorbid diabetes mellitus $(35.7 \%$; $\mathrm{n}=5 / 14)$ with depression. Majority of participants who had comorbid diabetes mellitus $72.7 \%(n=8)$ had onset $\leq 3$ years after CVD illness. They had more depression $(71.4 \%)$ as compared to those who had $>3$ years of duration of comorbid DM after the onset of CVD illness. 
Table 2 Psychosocial and behavioral related characteristics of the respondents in Jimma University Teaching Hospital cardiac clinic, 2014-2015 $(n=284)$

\begin{tabular}{|c|c|c|c|}
\hline Characteristics & $\begin{array}{l}\text { Non depressed } \\
\mathrm{N}(\%)\end{array}$ & $\begin{array}{l}\text { Depressed } \\
N(\%)\end{array}$ & $\begin{array}{l}\text { Total } \\
\text { N (\%) }\end{array}$ \\
\hline \multicolumn{4}{|l|}{ Living condition } \\
\hline Alone & $6(4.5)$ & $12(8.0)$ & $18(6.3)$ \\
\hline With family & $121(90.3)$ & $136(90.7)$ & $257(90.5)$ \\
\hline Other ${ }^{a}$ & $7(5.2)$ & $2(1.3)$ & $9(3.2)$ \\
\hline \multicolumn{4}{|c|}{ Oslo 3-items social support scale } \\
\hline Poor support & $38(28.4)$ & $62(41.3)$ & $100(35.2)$ \\
\hline Moderate support & $62(46.3)$ & $53(35.3)$ & $115(40.5)$ \\
\hline Strong support & $34(25.4)$ & $35(23.3)$ & $69(24.3)$ \\
\hline \multicolumn{4}{|l|}{ Physical activity } \\
\hline Yes & $79(59.0)$ & $66(44.0)$ & $145(51.1)$ \\
\hline No & $55(41.0)$ & $84(56.0)$ & $139(48.9)$ \\
\hline \multicolumn{4}{|c|}{ Life time used substances } \\
\hline \multicolumn{4}{|l|}{ Cigarette } \\
\hline Yes & $9(6.7)$ & $17(11.3)$ & $26(9.2)$ \\
\hline No & $125(93.3)$ & $133(88.7)$ & $258(90.8)$ \\
\hline \multicolumn{4}{|l|}{ Alcohol } \\
\hline Yes & $19(14.2)$ & $15(10.0)$ & $34(12.0)$ \\
\hline No & $115(85.8)$ & $135(90.0)$ & $250(88.0)$ \\
\hline \multicolumn{4}{|l|}{ Khat } \\
\hline Yes & $33(24.6)$ & $43(28.7)$ & $76(26.8)$ \\
\hline No & $101(75.4)$ & $107(71.3)$ & $208(73.2)$ \\
\hline \multicolumn{4}{|l|}{ Cannabis } \\
\hline Yes & $4(3.0)$ & $6(4.0)$ & $10(3.5)$ \\
\hline No & $130(97.0)$ & $144(96.0)$ & $274(96.5)$ \\
\hline \multicolumn{4}{|c|}{ Current used substances } \\
\hline \multicolumn{4}{|l|}{ Cigarette } \\
\hline Yes & $3(2.2)$ & $11(7.3)$ & $14(4.9)$ \\
\hline No & $131(97.8)$ & $139(92.7)$ & $270(95.1)$ \\
\hline \multicolumn{4}{|l|}{ Alcohol } \\
\hline Yes & $7(5.2)$ & $11(7.3)$ & $18(6.3)$ \\
\hline No & $127(94.8)$ & $139(92.7)$ & $266(93.7)$ \\
\hline \multicolumn{4}{|l|}{ Khat } \\
\hline Yes & $14(10.4)$ & $11(7.3)$ & $25(8.8)$ \\
\hline No & $120(89.6)$ & $139(92.7)$ & $259(91.2)$ \\
\hline \multicolumn{4}{|l|}{ Cannabis } \\
\hline Yes & $2(1.5)$ & $3(2.0)$ & $5(1.8)$ \\
\hline No & $132(98.5)$ & $147(98.0)$ & $279(98.2)$ \\
\hline
\end{tabular}

Living condition other ${ }^{\mathrm{a}}$ With relative/friends/homeless

Participants those who had comorbid hypertension constituted 45.1\% $(n=128)$. Among CVD patients with comorbid hypertension, depression prevalence was $46.0 \%(n=69)$. Among those who had comorbid hypertension their duration of onset $\leq 3$ years, after CVD illness $64.0 \%(n=33)$ were more depressed as compared to duration $>3$ years of comorbid hypertension. Majority of
Table 3 Depression relation to medication taken by CVD patients, JUTH cardiac clinic, 2014-2015 $(n=284)$

\begin{tabular}{|c|c|c|c|}
\hline Characteristics & $\begin{array}{l}\text { Total } \\
\text { N (\%) }\end{array}$ & $\begin{array}{l}\text { Nondepressed } \\
\text { N (\%) }\end{array}$ & $\begin{array}{l}\text { Depressed } \\
\text { N (\%) }\end{array}$ \\
\hline \multicolumn{4}{|l|}{ Medication } \\
\hline Digoxin & $62(21.8)$ & $33(24.6)$ & $29(19.3)$ \\
\hline Diuretic & $213(75.0)$ & $97(72.4)$ & $116(77.3)$ \\
\hline Beta blocker & $137(45.2)$ & $72(53.7)$ & $65(43.3)$ \\
\hline $\begin{array}{l}\text { Angiotensin-converting } \\
\text { enzyme inhibitor }\end{array}$ & $157(55.3)$ & $72(53.7)$ & $85(56.7)$ \\
\hline Calcium channel blocker & $24(8.5)$ & $9(6.7)$ & $15(10.0)$ \\
\hline Anticoagulant & $149(52.5)$ & $70(52.2)$ & $79(52.7)$ \\
\hline $\begin{array}{l}\text { Statine (lipid lowering } \\
\text { agents) }\end{array}$ & $22(7.7)$ & $11(8.2)$ & $11(7.3)$ \\
\hline Penicillin & $23(8.1)$ & $10(7.5)$ & $13(8.7)$ \\
\hline Other? & $17(6.0)$ & $8(6.0$ & $9(6.0)$ \\
\hline \multicolumn{4}{|l|}{ Duration of treatment (year) } \\
\hline$\leq 1$ & $91(32.0)$ & $50(37.3)$ & $41(27.3)$ \\
\hline $1-2$ & $51(18.0)$ & $24(17.9)$ & $27(18.0)$ \\
\hline $2-3$ & $29(10.2)$ & $12(9.0)$ & $17(11.3)$ \\
\hline $3-4$ & $30(10.6)$ & $11(8.2)$ & $19(12.7)$ \\
\hline$\geq 5$ & $83(29.2)$ & $37(27.6)$ & $46(30.7)$ \\
\hline \multicolumn{4}{|l|}{ Medication adherence } \\
\hline Non adherent & $78(27.5)$ & $45(30.0)$ & $33(24.6)$ \\
\hline Adherent & $206(72.5)$ & $105(70.0)$ & $101(75.4)$ \\
\hline
\end{tabular}

Other medications-vasodilator, broncho-dilator, steroid, PTU, insulin, metformine, daonil, valsartan

the participants $60.9 \%(n=173)$ with the previous history of hospitalization had more prevalence rate of depression (64.0\%), as compared to previously none hospitalized for CVD patients (Table 4).

New York Heart Association classification among CVD patients and its association with depression One-half of the participants (51.8\%) belonged to NYHA-class II as documented in the secondary data. The prevalence rate of depression in this group was $51.2 \%(n=62)$. This is more than in each of the remaining groups (Table 4).

Lipid profile among CVD patients and its association to depression Cholesterol was investigated and found that patients with a total cholesterol level of $<200 \mathrm{mg} /$ $\mathrm{dl}$ constituted in $78 \%$ and that they were more depressed 91.7\% as to compared with those having total cholesterol level $>200 \mathrm{mg} / \mathrm{dl}$. Those having lipoprotein level $>40 \mathrm{mg} /$ $\mathrm{dl}$ were $64 \%$ and they were the ones more depressed as compared to those whose LDL level of was $<40 \mathrm{mg} /$ dl. Similarly CVD patients with triglycerides level of $<150 \mathrm{mg} / \mathrm{dl}$ were compared with having triglycerides level $>150 \mathrm{mg} / \mathrm{dl}$ but there was no statistically significant difference in the level of depression (Table 4). 
Table 4 Distribution of respondents by clinical related factors in JUTH cardiac clinic, 2014-2015 $(n=284)$

\begin{tabular}{|c|c|c|c|}
\hline Characteristics & $\begin{array}{l}\text { Total } \\
\mathrm{N}(\%)\end{array}$ & $\begin{array}{l}\text { No depressed } \\
\mathrm{N} \%\end{array}$ & $\begin{array}{l}\text { Depressed } \\
\text { N (\%) }\end{array}$ \\
\hline \multicolumn{4}{|l|}{ Diagnosis type of CVD illness } \\
\hline Hypertensive heart diseases & $102(35.9)$ & $48(35.8)$ & $54(36.0)$ \\
\hline Ischemic heart disease & $75(26.4)$ & $37(27.6)$ & $38(25.3)$ \\
\hline Valvular heart disease & $35(12.3)$ & $11(8.2)$ & $24(16.0)$ \\
\hline Rheumatic heart disease & $26(9.2)$ & $14(10.4)$ & $12(8.0)$ \\
\hline Cardiomyopathy & $29(10.2)$ & $16(11.9)$ & $13(8.7)$ \\
\hline Other & $17(5.0)$ & $8(6.0)$ & $9(6.0)$ \\
\hline \multicolumn{4}{|l|}{ Duration of CVD illness (year) } \\
\hline$\leq 1$ & $68(23.9)$ & $36(26.9)$ & $32(21.3)$ \\
\hline $1-3$ & $78(27.5)$ & $37(27.6)$ & $41(27.3)$ \\
\hline $3-5$ & $58(20.4)$ & $26(19.4)$ & $32(21.3)$ \\
\hline $5-7$ & $35(12.3)$ & $15(11.2)$ & $20(13.3)$ \\
\hline$>7$ & $45(15.8)$ & $20(14.9)$ & $25(16.7)$ \\
\hline \multicolumn{4}{|l|}{ Family history of mental illness } \\
\hline Yes & $36(12.7)$ & $16(11.9)$ & $20(13.3)$ \\
\hline No & $248(87.3)$ & $118(88.1)$ & $130(86.7)$ \\
\hline \multicolumn{4}{|l|}{ Comorbid diabetes mellitus } \\
\hline Yes & $14(4.9)$ & $5(3.7)$ & $9(6.0)$ \\
\hline No & $270(95.1)$ & $129(96.3)$ & $141(94.0)$ \\
\hline \multicolumn{4}{|c|}{ Duration of DM after onset of CVD illness (year) } \\
\hline$\leq 3$ & $8(72.7)$ & $3(75.0)$ & $5(71.4)$ \\
\hline$>3$ & $3(27.3)$ & $1(25.0)$ & $2(28.6)$ \\
\hline \multicolumn{4}{|l|}{ Comorbid hypertension } \\
\hline Yes & $128(45.1)$ & $59(44.0)$ & $69(46.0)$ \\
\hline No & $156(54.9)$ & $75(56.0)$ & $81(54.0)$ \\
\hline \multicolumn{4}{|c|}{ Duration of HTN after onset of CVD illness (years) } \\
\hline$\leq 3$ & $60(63.8)$ & $27(62.8)$ & $33(64.7)$ \\
\hline$>3$ & $34(36.2)$ & $16(37.2)$ & $18(35.3)$ \\
\hline \multicolumn{4}{|l|}{ NYHA class } \\
\hline । & $16(7.1)$ & $6(5.8)$ & $10(8.3)$ \\
\hline$\|$ & $116(51.8)$ & $54(52.4)$ & $62(51.2)$ \\
\hline III & $57(25.4)$ & $27(26.2)$ & $30(24.8)$ \\
\hline IV & $35(15.6)$ & $16(15.5)$ & $19(15.7)$ \\
\hline \multicolumn{4}{|l|}{ Lipid profile level } \\
\hline \multicolumn{4}{|l|}{ Total cholesterol (mg/dl) } \\
\hline$<200$ & $18(78.3)$ & $7(63.6)$ & $11(91.7)$ \\
\hline$\geq 200$ & $5(21.7)$ & $4(36.4)$ & $1(8.3)$ \\
\hline \multicolumn{4}{|l|}{ Low density lipoprotein (mg/dl) } \\
\hline$<40$ & $9(36.0)$ & $4(36.4)$ & $5(35.7)$ \\
\hline$\geq 40$ & $16(64.0)$ & $7(63.6)$ & $9(64.3)$ \\
\hline \multicolumn{4}{|l|}{ Triglycerides (mg/dl) } \\
\hline$<150$ & $10(45.5)$ & $4(40.0)$ & $6(50.0)$ \\
\hline$\geq 150$ & $12(54.5)$ & $6(60.0)$ & $6(50.0)$ \\
\hline
\end{tabular}

Other diagnosis-corpulmonary, acute rheumatic fever, congenital heart disease, pericarditis, CHF due to thyrotoxicosis

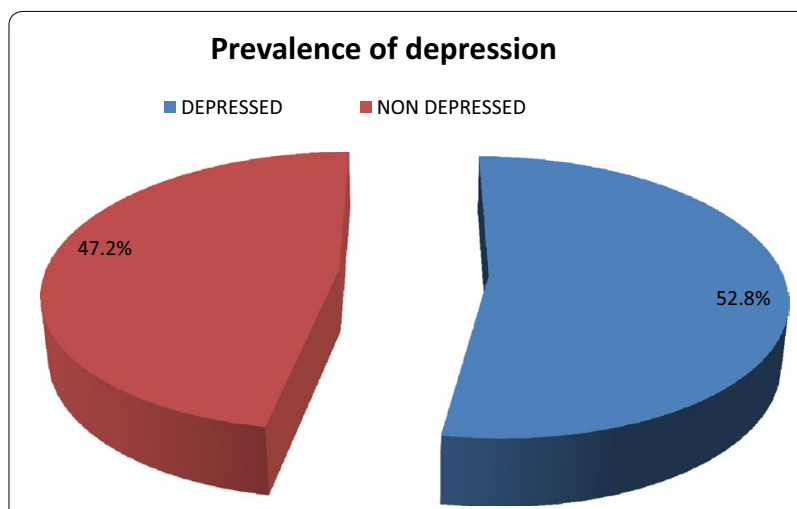

Fig. 1 Prevalence of depression among CVD patients in JUTH cardiac clinic, 2014-2015 ( $n=284)$. PHQ-9 classification of Depression in Jimma University Teaching Hospital cardiac clinic, 2014/15 ( $n=284)$

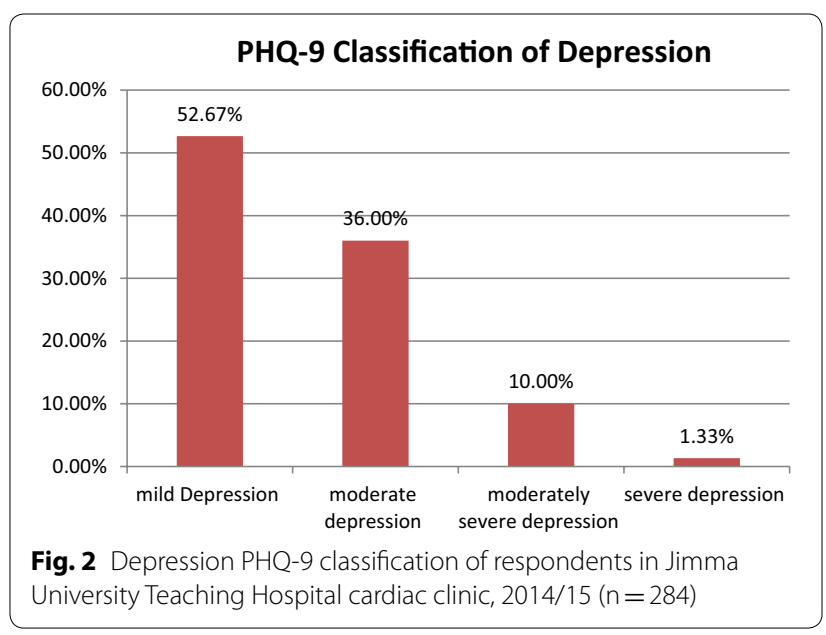

\section{Prevalence of depression}

Two hundred eighty-four of participants completed the interview of PHQ-9 questions at baseline. The prevalence of depression was $52.8 \%(n=150)$ (Fig. 1).

Out of the subjects with depression $52.67 \%(\mathrm{n}=79)$, $36.0 \%(n=54), 10.0 \%(n=15), 1.33 \%(n=2)$ mild, moderate, moderately severe, and severe depression, respectively had depressive symptoms (Fig. 2).

\section{Factors associated with depression in bivariate analysis among adult CVD patients}

Among different variables age, marital status, educational status, employment status, residence, average annual income (according to quartile income classification), living condition, social support, physical activity, current 
Table 5 Socio-demographic factors that associated with depression in bivariate analysis (<.25) among CVD patients in Jimma University Teaching Hospital cardiac clinic, 2014-2015

\begin{tabular}{|c|c|c|c|c|}
\hline Characteristics & $\begin{array}{l}\text { Non depressed } \\
\mathrm{N}(\%)\end{array}$ & $\begin{array}{l}\text { Depressed } \\
\text { N (\%) }\end{array}$ & COR $(95 \% \mathrm{Cl})$ & P-value \\
\hline \multicolumn{5}{|l|}{ Age } \\
\hline $18-26$ & $17(12.7)$ & $17(11.3)$ & $1.077(.456,2.541)$ & .866 \\
\hline $27-35$ & $15(11.2)$ & $21(14.0)$ & $1.508(.644,3.531)$ & .344 \\
\hline $36-44$ & $20(14.9)$ & $13(8.7)$ & $.700(.291,1.686)$ & .426 \\
\hline $45-53$ & $22(16.4)$ & $25(16.7)$ & $1.224(.559,2.678)$ & .613 \\
\hline $54-62$ & $28(20.9)$ & $26(17.3)$ & 1 & \\
\hline $63-71$ & $22(16.4)$ & $28(18.7)$ & $1.371(.633,2.968)$ & .424 \\
\hline$\geq 72$ & $10(7.5)$ & $20(13.3)$ & $2.154(.852,5.448)$ & $.105^{\mathrm{a}}$ \\
\hline \multicolumn{5}{|l|}{ Marital status } \\
\hline Single & $20(14.9)$ & $13(8.7)$ & $.615(.291,1.298)$ & $.202^{\mathrm{a}}$ \\
\hline Married & $105(78.4)$ & $111(74.0)$ & 1 & \\
\hline Divorced/separate & $4(3.0)$ & $10(6.7)$ & $2.365(.720,7.772)$ & $.156^{\mathrm{a}}$ \\
\hline Widowed & $5(3.7)$ & $16(10.7)$ & $3.027(1.071,8.556)$ & $.037^{\mathrm{a}}$ \\
\hline \multicolumn{5}{|l|}{ Educational status } \\
\hline Illiterate & $67(50.0)$ & $84(56.0)$ & 1 & \\
\hline Able to read and write only & $20(14.9)$ & $36(24.0)$ & $1.436(.762,2.706)$ & .263 \\
\hline Primary (1-8) & $28(20.9)$ & $19(12.7)$ & $.541(.278,1.053)$ & $.070^{\mathrm{a}}$ \\
\hline Secondary (9-12) & $14(10.4)$ & $7(4.7)$ & $.399(.152,1.044)$ & $.061^{\mathrm{a}}$ \\
\hline Tertiary $(+12)$ & $5(3.7)$ & $4(2.7)$ & $.638(.165,2.470)$ & .515 \\
\hline \multicolumn{5}{|l|}{ Employment status } \\
\hline Unemployed & $16(11.9)$ & $35(23.3)$ & $2.069(1.053,4.067)$ & $.035^{\mathrm{a}}$ \\
\hline Employed & $12(9.0)$ & $4(2.7)$ & $.315(.097,1.024)$ & $.055^{\mathrm{a}}$ \\
\hline Farmer & $70(52.2)$ & $74(49.3)$ & 1 & \\
\hline Merchant & $9(6.7)$ & $10(6.7)$ & $1.051(.403,2.740)$ & 919 \\
\hline Retired & $7(5.2)$ & $5(3.3)$ & $.676(.205,2.228)$ & .52 \\
\hline Housewife & $11(8.2)$ & $14(9.3)$ & $1.204(.512,2.830)$ & .67 \\
\hline Other & $9(6.7)$ & $8(5.3)$ & $.841(.307,2.301)$ & .736 \\
\hline \multicolumn{5}{|l|}{ Residence } \\
\hline Rural areas & $80(59.7)$ & $103(68.7)$ & 1 & \\
\hline Urban areas & $54(40.3)$ & $47(31.3)$ & $.676(.415,1.101)$ & $.116^{\mathrm{a}}$ \\
\hline \multicolumn{5}{|l|}{ Average annual income } \\
\hline 0-999 & $33(24.6)$ & $37(24.7)$ & $1.393(.723,2.684)$ & .322 \\
\hline 1000-3599 & $32(23.9)$ & $37(24.7)$ & $1.437(.743,2.776)$ & .281 \\
\hline $3600-10,799$ & $28(20.9)$ & $43(28.7)$ & $1.908(.985,3.694)$ & $.055^{\mathrm{a}}$ \\
\hline$\geq 10,800$ & $41(30.6)$ & $33(22.0)$ & 1 & \\
\hline
\end{tabular}

a Variables which shown statistically significant association during the bivariate analysis

cigarette use, diagnosis type of CVD illness, age onset of CVD, B-blocker medication, duration of treatment for CVD and lipid profile level-total cholesterol were showed association with depression at $\mathrm{P}$-value $<.25$ on bivariate analysis (Tables 4, 5 and 6).

Those variables widowed, unemployed, poor social support, physical inactivity was significantly associated with depression in bivariate analysis at P-value $<.05$ (Tables 5, 6 and 7).
Factors associated with depression in multiple logistic regression among patients with CVD

Multivariable analysis was done and found that those who were unemployed had about 2 times the odds of developing depression as compared to farmers, adjusted odds ratio $(\mathrm{AOR}=2.248,1.102,4.583)$. The odds of developing depression among patients employed were $75 \%$ less as compared to farmers $(\mathrm{AOR}=.252, .073$, .869). The odds of developing depression among those 
Table 6 Psychosocial and behavioral related factors that associated with depression in bivariate analysis (<.25) among CVD patients in Jimma University Teaching Hospital cardiac clinic, 2014-2015

\begin{tabular}{|c|c|c|c|c|}
\hline Characteristics & $\begin{array}{l}\text { Non depressed } \\
\text { N (\%) }\end{array}$ & $\begin{array}{l}\text { Depressed } \\
\text { N (\%) }\end{array}$ & COR $(95 \% \mathrm{Cl})$ & P-value \\
\hline \multicolumn{5}{|l|}{ Living condition } \\
\hline Alone & $6(4.5)$ & $12(8.0)$ & $1.779(.648,4.886)$ & .263 \\
\hline With family & $121(90.3)$ & $136(90.7)$ & 1 & \\
\hline Other $^{\mathrm{a}}$ & $7(5.2)$ & $2(1.3)$ & $.254(.052,1.247)$ & $.091^{b}$ \\
\hline \multicolumn{5}{|c|}{ Oslo 3-items social support scale } \\
\hline Poor support & $38(28.4)$ & $62(41.3)$ & $1.909(1.106,3.293)$ & $.020^{\mathrm{b}}$ \\
\hline Moderate support & $62(46.3)$ & $53(35.3)$ & 1 & \\
\hline Strong support & $34(25.4)$ & $35(23.3)$ & $1.204(.662,2.189)$ & .542 \\
\hline \multicolumn{5}{|l|}{ Physical activity } \\
\hline Yes & $79(59.0)$ & $66(44.0)$ & $.547(.341, .877)$ & $.012^{\mathrm{b}}$ \\
\hline No & $55(41.0)$ & $84(56.0)$ & 1 & \\
\hline \multicolumn{5}{|l|}{ Cigarette current user } \\
\hline Yes & $3(2.2)$ & $11(7.3)$ & $3.456(.943,12.664)$ & $.061^{\mathrm{b}}$ \\
\hline No & $131(97.8)$ & $139(92.7)$ & 1 & \\
\hline
\end{tabular}

Living condition other ${ }^{\mathrm{a}}$ with relative/friends/homeless

${ }^{\mathrm{b}}$ Variables which shown statistically significant association during the bivariate analysis

Table 7 Clinical and medication related factors that associated with depression in bivariate analysis (<.25) among CVD patients in Jimma University Teaching Hospital cardiac clinic, 2014-2015

\begin{tabular}{|c|c|c|c|c|}
\hline Characteristics & $\begin{array}{l}\text { Non depressed } \\
\mathrm{N}(\%)\end{array}$ & $\begin{array}{l}\text { Depressed } \\
\text { N (\%) }\end{array}$ & $\operatorname{COR}(95 \% \mathrm{Cl})$ & P-value \\
\hline \multicolumn{5}{|l|}{ Diagnosis type of CVD illness } \\
\hline Hypertensive heart diseases & $102(35.9)$ & $48(35.8)$ & 1 & \\
\hline Ischemic heart disease & $75(26.4)$ & $37(27.6)$ & $.913(.503,1.658)$ & .765 \\
\hline Valvular heart disease & $35(12.3)$ & $11(8.2)$ & $1.939(.86,4.371)$ & $.110^{\mathrm{a}}$ \\
\hline Rheumatic heart disease & $26(9.2)$ & $14(10.4)$ & $.762(.321,1.807)$ & .537 \\
\hline Cardiomyopathy & $29(10.2)$ & $16(11.9)$ & $.722(.315,1.654)$ & .442 \\
\hline Other & $17(5.0)$ & $8(6.0)$ & $1.000(.357,2.797)$ & 1.00 \\
\hline \multicolumn{5}{|l|}{ Age onset of CVD } \\
\hline $9-19$ & $11(8.2)$ & $12(8.0)$ & $.679(.264,1.745)$ & .421 \\
\hline $20-29$ & $17(12.7)$ & $21(14.0)$ & $.769(.347,1.701)$ & .516 \\
\hline $30-39$ & $24(17.9)$ & $19(12.7)$ & $.493(229,1.058)$ & $.070^{\mathrm{a}}$ \\
\hline $40-49$ & $24(17.9)$ & $27(18.0)$ & $.700(.339,1.445)$ & .335 \\
\hline $50-59$ & $30(22.4)$ & $26(17.3)$ & $.539(.266,1.092)$ & $.086^{\mathrm{a}}$ \\
\hline$\geq 60$ & $28(20.9)$ & $45(30.0)$ & 1 & 1 \\
\hline \multicolumn{5}{|l|}{ Medication } \\
\hline B-blocker & $72(53.7)$ & $65(43.3)$ & $1.519(.950,2.426)$ & $.081^{\mathrm{a}}$ \\
\hline \multicolumn{5}{|l|}{ Duration of treatment (year) } \\
\hline$\leq 1$ & $91(32.0)$ & $50(37.3)$ & 1 & \\
\hline $1-2$ & $51(18.0)$ & $24(17.9)$ & $1.372(.69,2.729)$ & .367 \\
\hline $2-3$ & $29(10.2)$ & $12(9.0)$ & $1.728(.741,4.028)$ & $.206^{\mathrm{a}}$ \\
\hline $3-4$ & $30(10.6)$ & $11(8.2)$ & $2.106(.901,4.927)$ & $.086^{\mathrm{a}}$ \\
\hline$\geq 5$ & $83(29.2)$ & $37(27.6)$ & $1.516(.834,2.758)$ & $.173^{\mathrm{a}}$ \\
\hline \multicolumn{5}{|c|}{ Lipid profile level_-total cholesterol (mg/dl) } \\
\hline$<200$ & $7(63.6)$ & $11(91.7)$ & $4.488(.495,40.69)$ & $.182^{\mathrm{a}}$ \\
\hline$\geq 200$ & $4(36.4)$ & $1(8.3)$ & 1 & \\
\hline
\end{tabular}

Diagnosis of CVD other-corpulmonary, acute rheumatic fever, congenital heart disease, pericarditis, CHF due to thyrotoxicosis

a Variables which shown statistically significant association during the bivariate analysis 
Table 8 Factors that associated with depression in multiple logistic regression of CVD patients in Jimma University Teaching Hospital cardiac clinic, 2014-2015 $(\mathrm{n}=284)$

\begin{tabular}{|c|c|c|c|c|}
\hline Characteristics & $\begin{array}{l}\text { Non depression } \\
\mathrm{N}(\%)\end{array}$ & $\begin{array}{l}\text { Depression } \\
\mathrm{N}(\%)\end{array}$ & AOR $(95 \% \mathrm{Cl})$ & P-value \\
\hline \multicolumn{5}{|l|}{ Occupational status } \\
\hline Unemployed & $16(11.9)$ & $35(23.3)$ & $2.248(1.102,4.583)^{\mathrm{a}}$ & .026 \\
\hline Employed & $12(9.0)$ & $4(2.7)$ & $.252(.073, .869)^{\mathrm{a}}$ & .029 \\
\hline Farmer & $70(52.2)$ & $74(49.3)$ & 1 & \\
\hline Merchant & $9(6.7)$ & $10(6.7)$ & $.904(.326,2.510)$ & .847 \\
\hline Retired & $7(5.2)$ & $5(3.3)$ & $.723(.212,2.472)$ & .605 \\
\hline Housewife & $11(8.2)$ & $14(9.3)$ & $1.417(.587,3.419)$ & .438 \\
\hline Other & $9(6.7)$ & $8(5.3)$ & $.635(.224,1.805)$ & .395 \\
\hline \multicolumn{5}{|c|}{ Oslo 3-items social support scale } \\
\hline Poor support & $38(28.4)$ & $62(41.3)$ & $2.324(1.290,4.187)^{\mathrm{a}}$ & .005 \\
\hline Moderate support & $62(46.3)$ & $53(35.3)$ & 1 & \\
\hline Strong support & $34(25.4)$ & $35(23.3)$ & $1.336(.702,2.545)$ & .378 \\
\hline \multicolumn{5}{|l|}{ Current cigarette user } \\
\hline Yes & $3(2.2)$ & $11(7.3)$ & $4.722(1.218,18.311)^{\mathrm{a}}$ & .025 \\
\hline No & $131(97.8)$ & $139(92.7)$ & 1 & \\
\hline \multicolumn{5}{|l|}{ Physical activity } \\
\hline Yes & $79(59.0)$ & $66(44.0)$ & $.541(.328, .893)^{\mathrm{a}}$ & .016 \\
\hline No & $55(41.0)$ & $84(56.0)$ & 1 & \\
\hline
\end{tabular}

a Significant level in $95 \% \mathrm{Cl}$

who had poor social support was 2 times odd of depression as compared to those with moderate social support $(\mathrm{AOR}=2.324,1.290,4.187)$. Similarly, among patients, current cigarette user were 5 times greater odds to develop depression as compared to those who were none current cigarette user $(\mathrm{AOR}=4.722,1.218,18.311)$. The odds of developing depression among patients who have a habit of doing physical activity was 53\% less as compared to who didn't do physical activity $(\mathrm{AOR}=.541$, .328, .893) (Table 8).

\section{Discussion}

In this study, an attempt was made to assess the prevalence and associated factors of depression. The prevalence of depression was $52.8 \%$ which high as compared to a study done in the US (21.5\%), Pakistan (37\%), and Jamaica (19.9\%) [19, 30, 31]. The prevalence of depression was almost similar to the study done in Washington, USA $51.0 \%$ [38]. However, it was lower in magnitude than a study done in Nigerian 67.0\% [50], in Brazil 67.0\% [32] and USA (Utah) (75\%) [27].

In our study, the finding of depression was higher than the studies done in Pakistan (37.0\% and 47.0\%), Netherlands (42\%) Iran (41.9\%), Canada (35\%) and Nigeria (30.0\%) [30, 33-37]. The difference might be studying population, study setting, the tool used, study design and sociocultural difference. The prevalence of depression was higher among CVD patients. This implies that doctors and other professionals should routinely screen depression among patients with CVD.

In this study CVD related employment status, physical activity, current cigarette user and social support were found the potent variable that statically significant association with depression in the final model.

Our study finding revealed that the likelihood of developing depression among those unemployed was 2 times higher than as compared to farmers $(\mathrm{AOR}=2.248,1.102$, 4.583). The possibility of developing depression among patients employed was $75 \%$ less likely as compared to farmers $(\mathrm{AOR}=.252, .073, .869)$. This study in line with the study done in South Korea and the USA [38, 39]. They are more likely to engage in drinking alcohol, smoking, drug use, suicide intentions, and crime. Additionally, because a majority of people among CVD patients were pre-retirement or retirement age was forced to leave their jobs. These things lead to depression among the unemployed [39]. Meta-analyses and systematic reviews did have shown that unemployed have at least twofold risk of mental illness, particularly depression and anxiety disorders, compared to employed persons [40].

In our finding, the likelihood of developing depression among those who had poor social support was 2 times odd of depression as compared to those with moderate social support $(\mathrm{AOR}=2.324,1.290,4.187)$. This finding 
similar to the study done in Canada, Italy, the USA, Brazil, and Iran [41-46]. Low social support levels are important risk factors for the subsequent development or worsening of depression.

In our study finding, among patients who current cigarette user was 5 times more likely to develop depression as compared to those who were none current cigarette user $(\mathrm{AOR}=4.722,1.218,18.311)$. This finding similar to the study done in the Netherlands supports that smoking as an independent predictor of depression in CVD patients. Smokers, in turn, have a higher risk of being depressed and a decreased chance of recovery from depression. Both tobacco smoking and depression are associated with cardiac mortality and morbidity [47].

Similarly, the study had done in Australia supports our study finding that reports, smoking as an independent predictor of depression in CVD patients. The mechanisms through which smoking is associated with depression might be biogenetic, psychological and environmental factors. Smoking-induced neurobiological changes that might predispose to depression; the transient alleviation of depressive symptoms and psychotropic side effects with smoking [48]. The study was done in Greece and systematic review done also reported that smokers have higher rates of depressive symptoms than nonsmokers $[49,50]$. Smoking cessation among depressed CVD patients is associated with concurrent improvement in depression [51]. Our study finding also contrasts with the study done in Toronto, Canada [52].

In our study participants, those who physically active (do moderate to vigorous activity for $30 \mathrm{~min}$ or more, at least 4 days in a week) were $46 \%$ less likely to develop depression as compared to physically inactivate, $(\mathrm{AOR}=.541, .328, .893)$. This is consistent with the study done in Italy and Spain that report that physical inactivity increases the risk of depression [53, 54]. The study was done in Pakistan also supports our study finding, additionally reported that physical activity shows positive effects to reduce the level of depression among CVD patients [55]. Other studies which were done in Canada and the USA also in line with our study finding [56]. Lastly, in this study respondent's age, sex, place of residence, B-blocker medication, marital status, comorbid diabetes mellitus and hypertension, living condition and lipid profile were not significantly associated with depression among CVD patients in the final model. The finding was in line with the study done in USA, Netherland, Japan, Denmark, Brazil, Pakistan [12, 20, 27, 30, 32, $38,53,57-59]$.

\section{Conclusions}

Our study finding showed that the prevalence of depression was $52.8 \%$ and it was found to be highly prevalent psychiatric comorbidity in adult CVD patients. Depression had independently associated with employment status, physical activity, current cigarette user and social support. This study could be taken as an eye-opener with regards to bridging the knowledge gap that exists in the country and particularly among Jimma University Teaching Hospital's health workers and specialists working with cardiac patients attending cardiac clinic and could help to put an effort for integration of knowledge from both Internists and psychiatrists through multidisciplinary team approach for achieving quality of patient care.

\section{Abbreviations}

ACS: acute coronary syndrome; AMl: acute myocardial infarction; AF: atrial fiberaltion; BDI: Beck depression inventory; BMI: body mass index; BSC: Bachelor of science; CAD: coronary arterial disease; CABG: coronary artery bypass graft; CHD: coronary heart disease; CHF: congestive heart disease; CVD: cardiovascular disease; CPMJU: College of Public Health Medical Science of Jimma University; DIS: diagnostic interview schedule; DSM-IV: Diagnostic and Statistical Manual of Mental Health Disorders, Fourth Edition; HADS: Hospital anxiety and depression scale; HHD: hypertension heart disease; HRQOL: heart-related quality of life; HTN: hypertension; ICD: implantable cardioverter-defibrillator; IHD: ischemic heart disease; JUST: Jimma University Specialized Hospital; JUTH: Jimma University Teaching Hospital; LVEF: left ventricular ejection fraction; MDD: major depressive disorder; MI: myocardial infarction; NYHA: New York Heart Association; NCDs: non-communicable diseases; PHQ-9: patient health questioner; QOL: quality of life; RHD: rheumatic heart disease; SHHD: Sudan household survey; SPSS: statistical package for social sciences; USA: United States of America; WHO: World health organization; YLL: years of life lost.

\section{Authors' contributions}

$H U, M B$, and $A B$ contributed to the design, conduct analysis of the research and in the manuscript preparation. AN contributed to the design, conduct, and analysis of the research. All authors read and approved the final manuscript.

\section{Author details}

${ }^{1}$ Research and Training Department of Amanuel Mental Specialized Hospital, Addis Abeba, Ethiopia. ${ }^{2}$ Department of Psychiatry, College of Public Health and Medical Sciences, Jimma University, Jimma, Ethiopia. ${ }^{3}$ Department of Psychiatry, College of Medicine and Health Sciences, Wollo University, Dessie, Ethiopia.

\section{Acknowledgements}

We would like to express our gratitude and sincere thanks to the Department of Psychiatry, College of Public Health and Medical Sciences of Jimma University for arranging this opportunity to carry out this research thesis. And also, our sincere and deepest thanks go to the Jimma University Teaching Hospital administration office to allow us to conduct this study. At last, our gratitude to study participants for their unlimited and valuable information and participated in this study.

\section{Competing interests}

The authors declare that they have no competing interests. 


\section{Consent for publication}

We all authors approve that our consent for publication our manuscript titled as prevalence and associated factors of depressive disorders among adult patients with cardiovascular disease at the outpatient cardiac clinic in Jimma University Teaching Hospital, southwest Ethiopia in the international journal of a mental health system. And we put our agreement signature as below.

\section{Name of Authors}

1. Halima Umer

2. Alemayehu Negash

3. Mengesha Birkie

4. Asmare Belete

\section{Signature}

$$
\text { बing } 11 / 20 / 2018
$$$$
\text { Annf }
$$$$
11-20-2018
$$

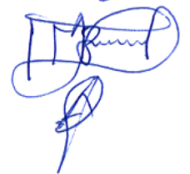

Lt/ $20 / 2018$

$11 / 20 / 2018$

\section{Data availability}

The authors approve that all data underlying the findings are completely accessible without limitation and also pertinent data are in the paper.

\section{Funding}

This is from a developing country; our salary couldn't cover the fee for publication. This paper is original and has a great impact on to design prevention strategy for policymaker and it will be used as the reference for researchers. Therefore, I request your journal to publish this paper free.

\section{Informed consent}

Procedures followed were in accordance with the ethical standards of the responsible committee from the Ethical Review Board of Jimma University, College of public health and Medical Sciences. Oral consent was obtained from the study participants. Patient those found to be severally depressed and suicidal risk appropriate intervention was done according to PHQ-9 score.

\section{Publisher's Note}

Springer Nature remains neutral with regard to jurisdictional claims in published maps and institutional affiliations.

Received: 27 November 2018 Accepted: 22 February 2019 Published online: 05 March 2019

\section{References}

1. Bloom DE, Cafiero E, Jané-Llopis E, Abrahams-Gessel S, Bloom LR, Fathima S, et al. The global economic burden of noncommunicable diseases. Program on the Global Demography of Aging. 2012.

2. Kessler RC, Ormel J, Petukhova M, McLaughlin KA, Green JG, Russo $L J$, et al. Development of lifetime comorbidity in the World Health Organization world mental health surveys. Arch Gen Psychiatry. 2011;68(1):90-100.

3. Funk M. Global burden of mental disorders and the need for a comprehensive, coordinated response from health and social sectors at the country level. 2016.

4. Gelaye B, Lemma S, Deyassa N, Bahretibeb Y, Tesfaye M, Berhane Y, et al. Prevalence and correlates of mental distress among working adults in Ethiopia. Clin Pract Epidemiol Ment Health CP EMH. 2012;8:126.

5. World Health Organization. Depression: a global crisis. World Mental Health Day, vol. 391. Geneva: World Health Organization; 2012.

6. Fuster V, Kelly BB. Committee on preventing the global epidemic of cardiovascular disease: meeting the challenges in developing countries board on global health. Kelly, editors. 2010.

7. Joseph P, Leong D, McKee M, Anand SS, Schwalm J-D, Teo K, et al. Reducing the global burden of cardiovascular disease, part 1: the epidemiology and risk factors. Circ Res. 2017;121(6):677-94.

8. Elderon L, Whooley MA. Depression and cardiovascular disease. Prog Cardiovasc Dis. 2013;55(6):511-23.
9. Khawaja IS, Westermeyer JJ, Gajwani P, Feinstein RE. Depression and coronary artery disease: the association, mechanisms, and therapeutic implications. Psychiatry. 2009;6(1):38.

10. Huffman JC, Celano CM, Beach SR, Motiwala SR, Januzzi JL. Depression and cardiac disease: epidemiology, mechanisms, and diagnosis. Cardiovasc Psychiatry Neurol. 2013. https://doi.org/10.1155/2013/695925.

11. Connerney I, Shapiro PA, MCLaughlin JS, Bagiella E, Sloan RP. Depression and outcome after coronary artery bypass surgery. Lancet. 2002;359(9317):1618.

12. Gottlieb SS, Khatta M, Friedmann E, Einbinder L, Katzen S, Baker B, et al. The influence of age, gender, and race on the prevalence of depression in heart failure patients. J Am Coll Cardiol. 2004;43(9):1542-9.

13. Kronish IM, Krupka DJ, Davidson KW. How should we treat depression in patients with cardiovascular disease? Dialogues Cardiovasc Med DCM. 2012;17(2):126.

14. Mallik S, Spertus JA, Reid KJ, Krumholz HM, Rumsfeld JS, Weintraub WS, et al. Depressive symptoms after acute myocardial infarction: evidence for highest rates in younger women. Arch Intern Med. 2006;166(8):876-83.

15. Strik JJ, Lousberg R, Cheriex EC, Honig A. One year cumulative incidence of depression following myocardial infarction and impact on the cardiac outcome. J Psychosom Res. 2004;56(1):59-66.

16. Lespérance F, Frasure-Smith N. Depression in patients with cardiac disease: a practical review. J Psychosom Res. 2000;48(4-5):379-91.

17. Suliman A. The state of heart disease in Sudan. Cardiovasc J Afr. 2011;22(4):191.

18. Tully PJ, Bennetts JS, Baker RA, McGavigan AD, Turnbull DA, Winefield HR. Anxiety, depression, and stress as risk factors for atrial fibrillation after cardiac surgery. Heart Lung J Acute Crit Care. 2011;40(1):4-11.

19. Rutledge T, Reis VA, Linke SE, Greenberg BH, Mills PJ. Depression in heart failure: a meta-analytic review of prevalence, intervention effects, and associations with clinical outcomes. J Am Coll Cardiol. 2006;48(8):1527-37.

20. Maharaj R, Reid S, Misir A, Simeon D. Depression and its associated factors among patients attending chronic disease clinics in southwest Trinidad. West Indian Med J. 2005;54(6):369-74.

21. Gafarov VV, Panov DO, Gromova EA, Gagulin IV, Gafarova AV. The influence of depression on risk development of acute cardiovascular diseases in the female population aged 25-64 in Russia. Int J Circumpolar Health. 2013;72(1):21223.

22. Saran R, Puri A, Agarwal M. Depression, and the heart. Indian Heart J. 2012;64(4):397-401.

23. Khan FM, Kulaksizoglu B, Cilingiroglu M. Depression, and coronary heart disease. Curr Atheroscler Rep. 2010;12(2):105-9.

24. Go AS, Mozaffarian D, Roger VL, Benjamin EJ, Berry JD, Blaha MJ, et al. Heart disease and stroke statistics - 2014 update: a report from the American Heart Association. Circulation. 2013. https://doi. org/10.1161/01.cir.0000441139.02102.80.

25. Carney RM, Freedland KE, Miller GE, Jaffe AS. Depression as a risk factor for cardiac mortality and morbidity: a review of potential mechanisms. J Psychosom Res. 2002;53(4):897-902.

26. Huntington EB. Memories of Lakeside Camp. 1937.

27. Geddes MS. Depression detection in Hospitalized cardiac patients. 2010.

28. Fadare J, Lawal M, Elegbede A, Joseph D, Ampitan B, Ayodele M. Medication adherence and patients satisfaction among psychiatric outpatients in a rural Nigerian Tertiary Healthcare Facility. J Psychiatry. 2014;17:125. https://doi.org/10.4172/Psychiatry.1000125:2.

29. Abiola T, Udofia O, Zakari M. Psychometric properties of the 3-item Oslo social support scale among clinical students of Bayero University Kano, Nigeria. Malays J Psychiatry. 2013;22(2):32-41.

30. Bokhari S, Samad A, Hanif S, Hadique S, Cheema M, Fazal M, et al. Prevalence of depression in patients with coronary artery disease in a tertiary care hospital in Pakistan. JPMA J Pak Med Assoc. 2002;52(9):436-9.

31. Martin J, Neita S, Gibson R. Depression among cardiovascular disease patients on a consultation-liaison service at a general hospital in Jamaica. West Indian Med J. 2012;61(5):499-503.

32. Pena FM, da Silva Soares J, Paiva BTC, Piracicaba MCT, Marins RM, Barcellos $A F$, et al. Sociodemographic factors and depressive symptoms in hospitalized patients with heart failure. Exp Clin Cardiol. 2010;15(2):e29. 
33. Dogar IA, Khawaja IS, Azeem MW, Awan H, Ayub A, lqbal J, et al. Prevalence and risk factors for depression and anxiety in hospitalized cardiac patients in Pakistan. Psychiatry. 2008;5(2):38.

34. Jaarsma T, Lesman-Leegte I, Hillege HL, Veeger NJ, Sanderman R, van Veldhuisen DJ, et al. Depression and the usefulness of a disease management program in heart failure: insights from the $\mathrm{COACH}$ (Coordinating study evaluating Outcomes of Advising and Counseling in Heart failure) study. J Am Coll Cardiol. 2010;55(17):1837-43.

35. Bayani B, Yousefi S, Bayani M, Shirmohammadi M, Alimoradi A, Falsoleiman $\mathrm{H}$, et al. Depression and anxiety in a cardiovascular outpatient clinic: a descriptive study. Iran J Psychiatry. 2011;6(3):125.

36. Lauzon C, Beck CA, Huynh T, Dion D, Racine N, Carignan S, et al. Depression and prognosis following hospital admission because of acute myocardial infarction. Can Med Assoc J. 2003;168(5):547-52.

37. Mbakwem AC, Aina OF. Comparative study of depression in hospitalized and stable heart failure patients in an urban Nigerian teaching hospital. Gen Hosp Psychiatry. 2008;30(5):435-40.

38. Freedland KE, Rich MW, Skala JA, Carney RM, Dávila-Román VG, Jaffe AS. Prevalence of depression in hospitalized patients with congestive heart failure. Psychosom Med. 2003:65(1):119-28.

39. Song EK, Lennie TA, Moser DK. Depressive symptoms increase the risk of rehospitalization in heart failure patients with preserved systolic function. J Clin Nurs. 2009;18(13):1871-7.

40. Herbig B, Dragano N, Angerer P. Health in the long-term unemployed. Deutsches Ärzteblatt Int. 2013;110(23-24):413.

41. Frasure-Smith N, Lesperance F. Coronary artery disease, depression and social support only the beginning. Oxford: Oxford University Press; 2000.

42. Frasure-Smith N, Lespérance F, Gravel G, Masson A, Juneau M, Talajic M, et al. Social support, depression, and mortality during the first year after myocardial infarction. Circulation. 2000;101(16):1919-24.

43. Compare A, Zarbo C, Manzoni GM, Castelnuovo G, Baldassari E, Bonardi A, et al. Social support, depression, and heart disease: a ten year literature review. Front Psychol. 2013;4:384.

44. Rollman BL, Belnap BH, LeMenager MS, Mazumdar S, Houck PR, Counihan PJ, et al. Telephone-delivered collaborative care for treating post-CABG depression: a randomized controlled trial. JAMA. 2009;302(19):2095-103.

45. Lino VT, Portela MC, Camacho LA, Atie S, Lima MJ. Assessment of social support and its association to depression, self-perceived health and chronic diseases in elderly individuals residing in an area of poverty and social vulnerability in Rio de Janeiro City, Brazil. PLOS ONE. 2013:8(8):e71712.

46. Khayyam-Nekouei Z, Neshatdoost H, Yousefy A, Sadeghi M, Manshaee G. Psychological factors and coronary heart disease. ARYA Atheroscler. 2013;9(1):102.
47. de Jonge P, Bos EH. Tobacco smoking and depression: time to move on to a new research paradigm in medicine? BMC Med. 2013;11(1):138.

48. Stafford $\mathrm{L}$, Berk M, Jackson HJ. Tobacco smoking predicts depression and poorer quality of life in heart disease. BMC Cardiovasc Disord. 2013;13(1):35.

49. Doyle F, Rohde D, Rutkowska A, Morgan K, Cousins G, McGee H. Systematic review and meta-analysis of the impact of depression on subsequent smoking cessation in patients with coronary heart disease: 1990 to 2013. Psychosom Med. 2014;76(1):44-57.

50. Lembke A, Johnson K, DeBattista C. Depression and smoking cessation: does the evidence support psychiatric practice? Neuropsychiatr Dis Treat. 2007;3(4):487.

51. Busch AM, Borrelli B, Leventhal AM. The relationship between smoking and depression post-acute coronary syndrome. Curr Cardiovasc Risk Rep. 2011;5(6):510.

52. Gravely-Witte S, Stewart DE, Suskin N, Grace SL. The association among depressive symptoms, smoking status and antidepressant use in cardiac outpatients. J Behav Med. 2009;32(5):478.

53. Larsen KK, Christensen B, Søndergaard J, Vestergaard M. Depressive symptoms and risk of new cardiovascular events or death in patients with myocardial infarction: a population-based longitudinal study examining health behaviors and health care interventions. PLOS ONE. 2013;8(9):e74393.

54. Guallar-Castillón P, del Mar Magariños-Losada M, Montoto-Otero C, Tabuenca Al, Rodríguez-Pascual C, Olcoz-Chiva M, et al. Prevalence of depression and associated medical and psychosocial factors in elderly hospitalized patients with heart failure in Spain. Rev Esp Cardiol. 2006;59(8):770-8 (English Edition)

55. Abidi STJ. Is Physical activity and effective tool to reduce depression after coronary artery event? - a systematic review. 2013.

56. Mammen G, Faulkner G. Physical activity and the prevention of depression: a systematic review of prospective studies. Am J Prev Med. 2013;45(5):649-57.

57. May HT, Horne BD, Carlquist JF, Sheng X, Joy E, Catinella AP. Depression after coronary artery disease is associated with heart failure. J Am Coll Cardiol. 2009:53(16):1440-7.

58. van Melle JP, Verbeek DE, van den Berg MP, Ormel J, van der Linde MR, de Jonge P. Beta-blockers and depression after myocardial infarction: a multicenter prospective study. J Am Coll Cardiol. 2006;48(11):2209-14.

59. Kato N, Kinugawa K, Shiga T, Hatano M, Takeda N, Imai Y, et al. Depressive symptoms are common and associated with adverse clinical outcomes in heart failure with reduced and preserved ejection fraction. J Cardiol. 2012;60(1):23-30.

\footnotetext{
Ready to submit your research? Choose BMC and benefit from:

- fast, convenient online submission

- thorough peer review by experienced researchers in your field

- rapid publication on acceptance

- support for research data, including large and complex data types

- gold Open Access which fosters wider collaboration and increased citations

- maximum visibility for your research: over 100M website views per year
}

At BMC, research is always in progress.

Learn more biomedcentral.com/submissions 